\title{
Cambio climático a nivel de nicho y caña de azúcar. I
}

Tamazula, Jalisco. México

\author{
Climate change at the niche level and sugarcane. I Tamazula, \\ Jalisco, Mexico
}

\author{
Lomelí-Sandoval, Nora Araceli; Muñoz-Orozco, Abel; Zúniga \\ González, Carlos Alberto; Hernandez, Alvaro Caballero; Vázquez \\ Montenegro, M.Sc. Ranses José; Editor Academico Dr. Angel sol- \\ Sanhez
}

\author{
Nora Araceli Lomelí-Sandoval \\ amunozo@colpos.mx \\ IREGEP, México \\ (iD) Abel Muñoz-Orozco \\ amunozo@colpos.mx \\ IREGEP, México \\ (iD) Carlos Alberto Zúniga González \\ czuniga@ct.unanleon.edu.ni \\ Universidad Nacional Autónoma de Nicaragua, León., \\ Nicaragua \\ Alvaro Caballero Hernandez \\ alvro_caballero@yahoo.es \\ Universidad Nacional Autónoma de Nicaragua, León., \\ Nicaragua \\ M.Sc. Ranses José Vázquez Montenegro \\ ranses.vazquez@insmet.cu \\ Instituto de Meteorología de Cuba., Cuba \\ Editor Academico Dr. Angel sol-Sanhez \\ Colegio de postgraduados, Mexico, México
}

Revista Iberoamericana de Bioeconomía y Cambio Climático

Universidad Nacional Autónoma de Nicaragua, León, Nicaragua

ISSN-e: 2410-7980

Periodicidad: Semestral

vol. 1, núm. 1, 2015

czuniga@ct.unanleon.edu.ni

Recepción: 15 Julio 2014

Aprobación: 12 Diciembre 2014

URL: http://portal.amelica.org/ameli/journal/394/3941748005/

DOI: https://doi.org/https:/10.5377/ribcc.v1i1.2148

Autor de correspondencia: amunozo@colpos.mx
Resumen: El presente trabajo tuvo como objetivo estudiar las variaciones relacionadas con la gran sequía invernal (GSI), la sequía intraestival (SI), la ocurrencia de heladas, golpes de calor y excesos de lluvia, aprovechando datos históricos disponibles de la localidad de Tamazula, Jalisco como un espacio dentro de un nicho; así mismo, comparar las variaciones antes y después de 1975, año tomado como referencia de la manifestación del último cambio climático según la Figura 2. Otro objetivo colateral fue vincular dicho desarrollo histórico con un experimento de variedades de caña realizado en 2011-2013 cerca de Tamazula, Jalisco.

Palabras clave: Cambio Climático, Modelos simulación, Nicho, Meteorología.

Abstract: The objective of this work was to study the variations related to the great winter drought (GSI), intra-summer drought (SI), frost occurrence, heat stroke and excess rainfall, taking advantage of historical data available from the town of Tamazula, Jalisco as a space within a niche; Likewise, compare the variations before and after 1975, the year taken as reference for the demonstration of the latest climate change according to Figure 2. Another collateral objective was to link said historical development with an experiment of cane varieties carried out in 2011-2013 near Tamazula, Jalisco

Keywords: Climate change, simulation models, Niche, Meteorology. 


\section{INTRODUCION}

México, es mega diverso en lo biológico, ecológico, humano y en la interacción genético

ambiental. Dentro de su patrimonio pose una riqueza de mecanismo de adaptación a los factores adversos y una amplia diversidad de agro sistemas que encierran combinaciones de plantas y estrategias para hacer frente a los cambios que el clima requiera (Muñoz, 2005; Muñoz, et al. 2009; Muñoz-Orozco, 2014).

En la mayor parte del país la precipitación ocurre en la segunda mitad del año con un periodo de escasa lluvia denominado Gran Sequía Invernal (GSI) que puede extenderse de octubre de un año a junio del siguiente (Figura 1); adicionalmente ocurre de manera imprevisible un periodo de sequía dentro del ciclo de lluvias denominado Sequía Intraestival (SI) (Mosiño y García, 1978) que puede durar de uno a cuatro meses (Reyna, 1970) y causa severas reducciones de rendimiento cuando coincide con las etapas de gametogénesis, de floración o inicio del llenado de grano. Por su parte las temperaturas bajas extremas pueden ocurrir en forma de heladas en altitudes arriba de los 1900 metros y las altas en forma de golpes de calor en altitudes abajo de los $1900 \mathrm{~m}$; los excesos de lluvia generan inundaciones y reducciones de oxígeno en los cultivos. Con el cambio climático estos factores abióticos adversos se tornan más frecuentes, intensos, extremosos e imprevisibles (Muñoz y Barraza, 1997; Muñoz, et al., 2012; Lomelí, et al. 2012). De ahí que se haga necesario ampliar o desarrollar en las plantas de cultivo, según el caso, no solo la resistencia a sequía, sino a heladas, calor y anoxia, entre otras y sea necesario precisar los fenómenos adversos para poder diseñar los tratamientos en los esquemas genotécnicos correspondientes.

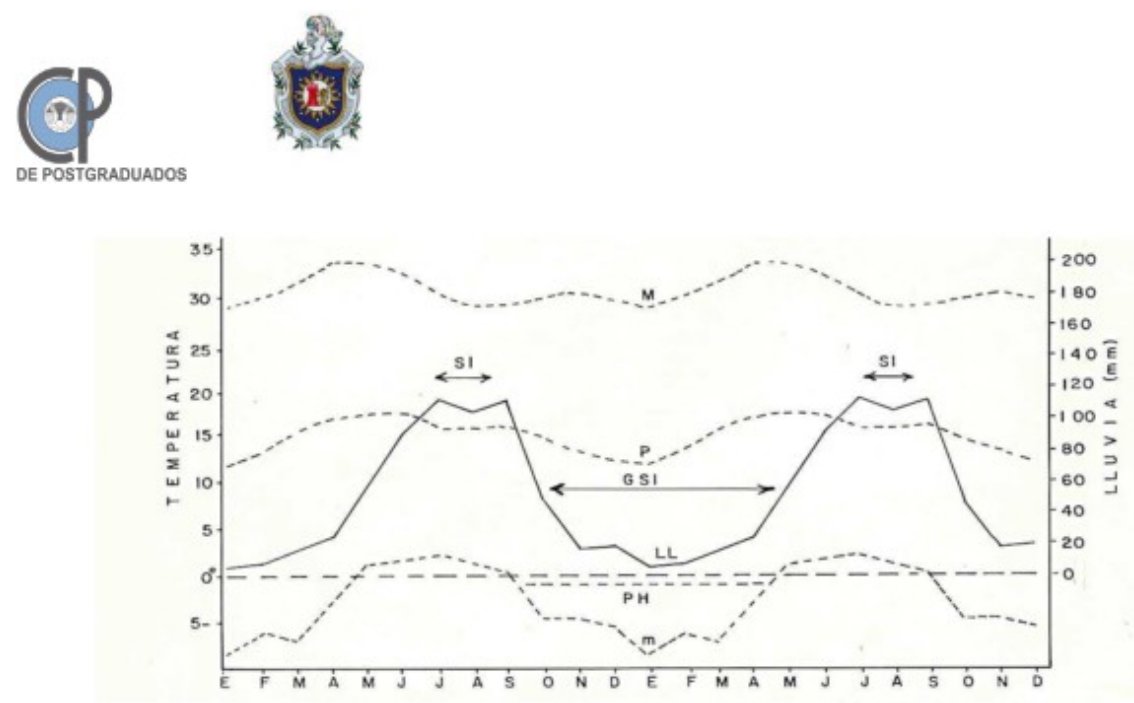

FIGURA 1.

Distribución de las temperaturas (oC) máximas (M), lluvia mensual (LL), temperaturas mínimas (m); al unir dos años se aprecia la Gran Sequía Invernal (GSI), el periodo de heladas (PH) y el descenso de lluvia que ocurre en esta localidad en agosto o Sequía Intraestival (SI), en Teotihucán, Estado de México, 19040'54.94” N, 98051'59.77” O, elevación 2277 m. Teotihuacán, Estado de México, 19040'54.94” N, 98051'59.77” O, elevación 2277 m.

Notas DE AUTOR 
Dentro de ese panorama mega diverso de México un rasgo importante es una orografía bastante irregular caracterizada por un mosaico de pequeños valles, micro regiones o nichos ecológicos que ocupan el $85 \%$ de su superficie. La agricultura en la mayor parte es de secano, llamada de temporal, y se desarrolla eminentemente teniendo como única fuente de agua la lluvia. Los trabajo sobre Cambio Climático Global o regional, basados en modelos aplicados a amplias regiones, si bien dan una orientación de las tendencias globales o regionales (IPCC, 2001), tienen poca utilidad en la escala micro regional, sobre todo en el campo agronómico y en especial en el mejoramiento genético de las resistencias. En donde es necesario tener precisos los niveles, duraciones y frecuencias de las variaciones meteorológicas asociadas a las resistencias que se trata de mejorar para diseñar las estrategias agronómicas o genotécnicas para hacer frente a los cambios que el clima valla exigiendo.

\section{OBJetivos}

El presente trabajo tuvo como objetivo estudian las variaciones relacionadas con la gran sequía invernal (GSI), la sequía intraestival (SI), la ocurrencia de heladas, golpes de calor y excesos de lluvia, aprovechando datos históricos disponibles de la localidad de Tamazula, Jalisco como un espacio dentro de un nicho; así mismo, comparar las variaciones antes y después de 1975, año tomado como referencia de la manifestación del último cambio climático según la Figura 2. Otro objetivo colateral fue vincular dicho desarrollo histórico con un experimento de variedades de caña realizado en 2011-2013 cerca de Tamazula, Jalisco.

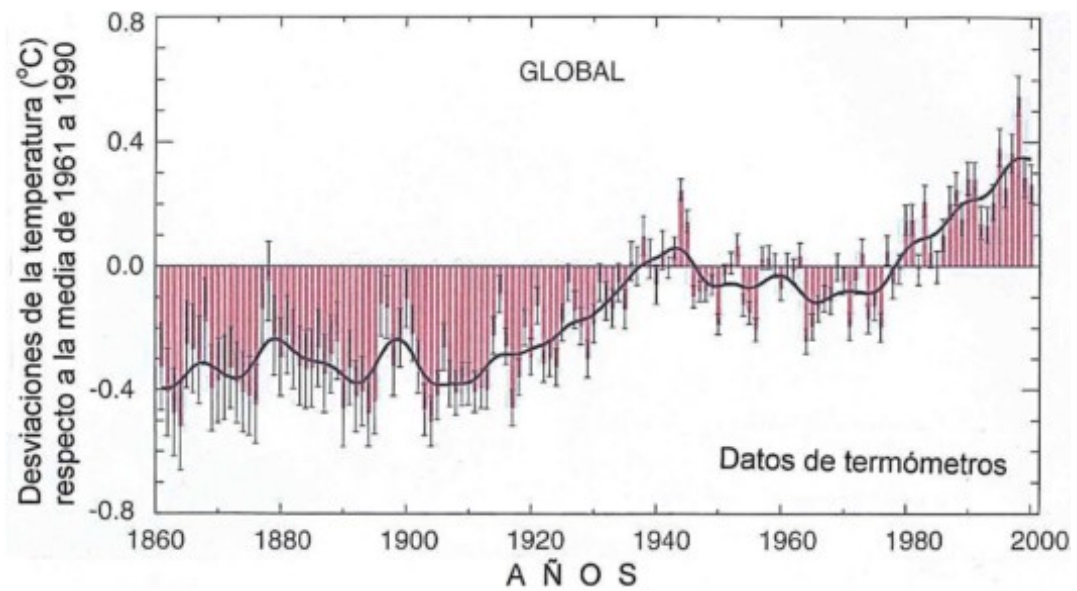

FIGURA 2.

Desviaciones de las temperaturas (oC) de la superficie del suelo y del mar combinadas, del periodo $1861-2000$ respecto al promedio del intervalo 1961-1990. Adaptación del autor en base a IPCC (2001, p 26) y con su anuencia. $\operatorname{IPCC}(2001)$

\section{Materiales y MÉtodos}

Tamazula, Jalisco se ubica en las coordenadas $103.23^{\circ}$ de longitud y $19.66^{\circ}$ de latitud a $1127 \mathrm{msnm}$.

Para los objetivos de este trabajo el tiempo con datos disponibles en la localidad de estudio, se dividió en dos periodos: uno de 1945 a 1974 (A1975) y dos de 1976-2005 (D1975), en base a que en forma global el incremento de temperatura en la época

reciente se manifiesta aproximadamente a partir de 1975 (Figura 2)

Las lluvias diarias de cada mes se sumaron en tres grupos, el primero de 10 días (día 1 a 10), el segundo también de 10 días (día 11 a 20) y el tercero según el mes (con 11, 8 o 10 días). Para el agrupamiento en 
10 días también se tomó como criterio el hecho que las plantas cultivadas empiezan presentar síntomas de deficiencias hídricas en textura ligeras después de 10 días sin agua, y que en riego se aplica agua entre los 10 y 20 días de no regar, y por otra parte que la lluvia diaria es demasiado dispersa y la mensual relativamente burda; de ahí que 10 días representen una precisión razonable. Para las heladas se consideró el periodo que va de octubre de un año a abril del siguiente y dentro de ese lapso se usó la mínima de las mínimas en cada decena; no se usó el promedio porque borra las variaciones pico en las cuales estamos interesados. Para los golpes de calor se consideró el periodo de abril a agosto dentro de cada año y dentro de ese lapso se usó la máxima de las máximas en cada decena con razonamiento similar al caso anterior. Para la Gran sequía invernal (GSI) abarcó de noviembre a mayo (Figura 1); sin embargo para saber si se está extendiendo hacía octubre y hacía junio se estudió la lluvia en el periodo que va de octubre de un año a junio del siguiente. La sequía intraestival (SI) es una reducción de lluvia que ocurre dentro del estío en un periodo de dos a cinco meses (Figura 3) (Reyna, 1970); para estudiarla, en Tamazula, Jalisco se abarcó el periodo de junio a octubre de cada año.

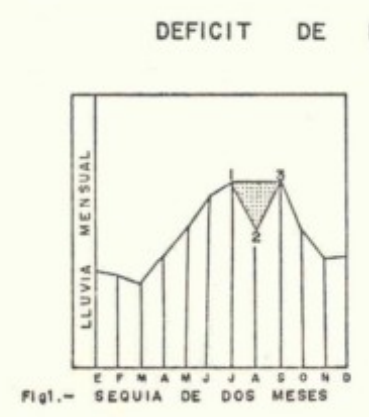

PRECIPITACION ESTIVAL
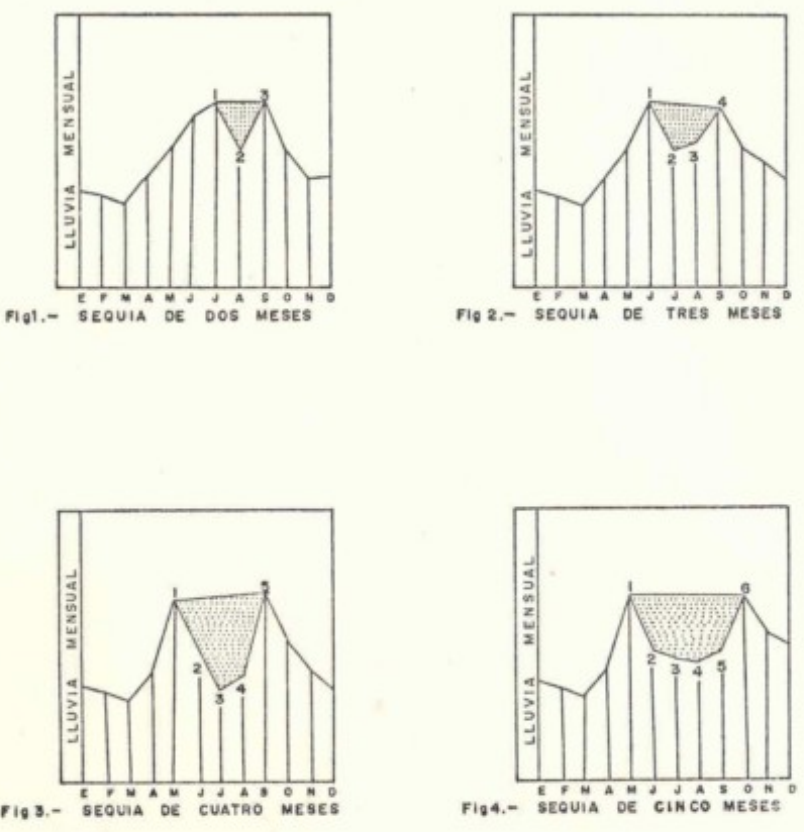

FIGURA 3.

déficits de la Sequía Intraestival (SI): Fig1 cuando es de dos meses; Fig 2 cuando es de tres meses; Fig 3 cuando es de cuatro meses; Fig 4 cuando es de cinco meses. Adaptación de los autores en base a la fuente: Reyna, (1970). Reyna, (1970)

De esa manera se tienen los siguientes factores de variación y niveles: Periodos (P) dos: antes de 1975 (A1975) y después de 1975 (D1975). Años (A): 1945-1974 (30 años); 1976-2005 (30 años) Meses (M), doce: $\mathrm{E}$, enero a $\mathrm{D}$, diciembre.

Decenas (D), tres: D1: días 1-10, D2: días 11-20, D3: días 21-último de cada mes. Con esos términos el modelo bajo un diseño completamente al azar es ec1:

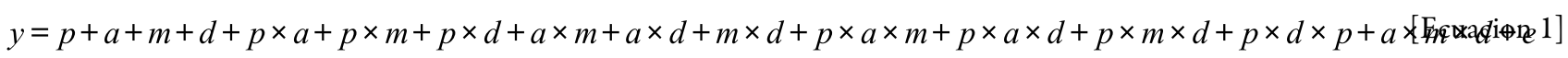


En donde Y es la variación total de temperaturas máximas semanales (Tmax), o de temperaturas mínimas semanales (Tmin), o de la lluvia durante la gran sequía invernal (LLGSI), o de la lluvia en la sequía intraestival (LLSI).

$\mathrm{P}$, representa la variación de los periodos; $\mathrm{A}$, la de los años; $\mathrm{M}$, la de los meses; $\mathrm{D}$, la de las decenas; los siguientes términos en forma de productos representan las variaciones de las diferentes interacciones que se generan entre los factores P, A, M y D. E, es la variación aleatoria en los análisis de variación de las variables en estudio: Tmax, Tmin, LLGSI y LLSI. El análisis de datos, se efectuó con el paquete SAS versión 9.0.

\section{RESULTADOS Y Discusión}

\section{TEMPERATURAS MAXIMAS DE LAS MAXIMAS DECENALES Interacción meses* periodos}

En esta microrregión (Tamazula, Jalisco) se apreció una disminución de las temperaturas máximas extremas entre febrero y junio de la primera mitad del año y un incremento entre agosto y noviembre de la segunda mitad del año (Figura 4). O sea que en este nicho el calentamiento global (Después de 1975) se dio en septiembre y octubre de la segunda mitad del año; en abril y mayo de la otra mitad se dio un enfriamiento. Esta inversión, puede relacionarse con el ambiente seco que predomina durante la GSI y la condición húmeda durante el verano-otoño.

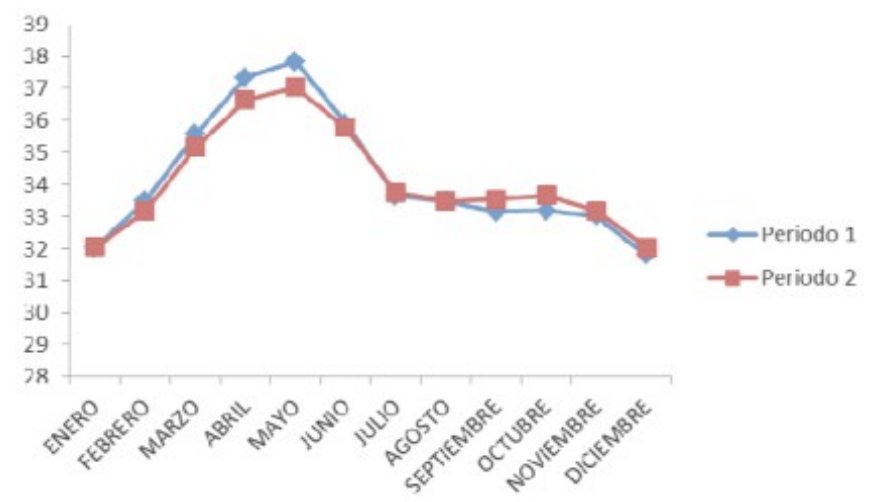

FIGURA 4.

Variación de la temperatura máxima en la interacción periodos*meses. Periodo 1, antes de 1975 y 2, después de 1975.

Figura 4.

\section{Interacción Meses*Decenas}

Según la Figura 5, de enero a marzo las terceras decenas se hicieron más calientes, las primeras decenas de enero a abril fueron más frescas respecto a las tercera; en mayo ocurrieron dos fenómenos: se alcanzó el máximo de los máximos y ocurrió una igualación de las tres decenas, continuando una inversión en junio; consecuentemente la primera decena se hizo más tibia y la tercera más fresca; después de esa inversión hubo un refrescamiento acompañado de una tendencia a igualación que perduró de julio a octubre. La dinámica de este proceso culminó con una inversión de las decenas de noviembre a diciembre respecto a la secuencia observada de enero a abril. Considerando los golpes de calor niveles de temperaturas arriba de $350 \mathrm{C}$, se infiere que estos pueden ocurrir de abril a mayo. En las tres decenas de diciembre hay un mes y en las tres de enero hay otro, quiere decir que en esos dos meses ocurre una igualación y una inversión. Resumiendo, entre las decenas 
de cada mes en cada ciclo anual ocurren dos igualaciones breves y una prolongada seguidas de una inversión; Tratándose de la caña en el occidente de México, los ascensos de la temperatura máxima como los de abril a mayo están asociados a baja humedad, en tales condiciones el ataque de barrenador se intensifica como lo observó Rodríguez (2013), queda pendiente precisarse en qué medida se afectan otras respuestas de la caña.

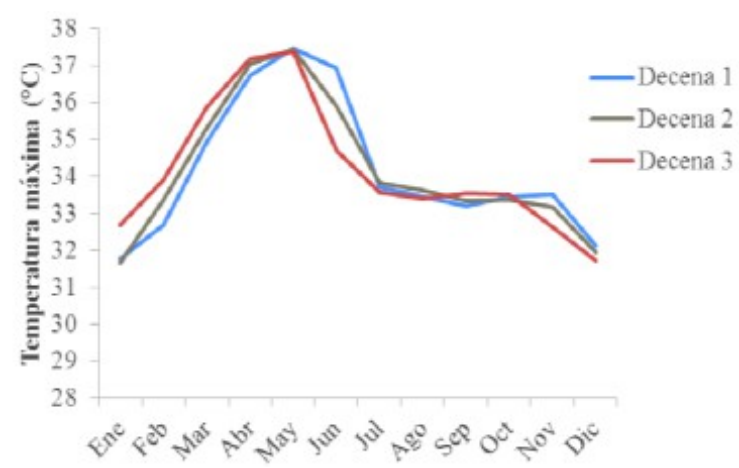

FIGURA 5.

Variación de las temperaturas máximas de las máximas decenales en la interacción mese*decenas. Decena 1: día 1 a 10; decena 2: dia11 a 20; decena 3: día 21 a día último del mes.

Figura 5.

\section{TEMPERATURAS MÍNIMAS DE LAS MÍNIMAS DECENALES Interacción periodos*años}

Esta interacción permite observar que en el nicho de Tamazula, Jalisco, de 1975 a 1990 hay un sutil incremento de la temperatura mínima de las mínimas decenales, que es más notorio después de 1990 (Figura 6). El que este fenómeno se de en las mínimas coincide con el hecho de que el calentamiento global se expresa en la noche cuando la radiación roja e infra roja predomina respecto al espectro que se recibe durante el día.

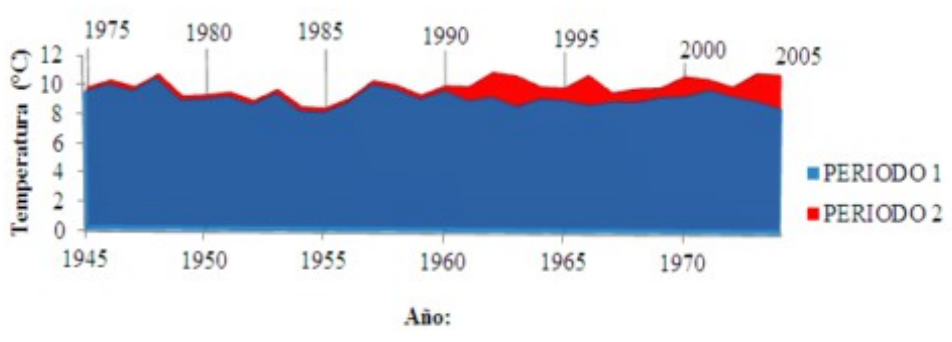

FIGURA 6

Incremento de las temperaturas mínimas en el periodo dos respecto al uno según la interacción periodos*años. figura 6.

Interacción periodos por meses sobre las temperaturas mínimas.

En esta interacción solo la mínima del periodo dos se aparta del paralelismo mediante un incremento en marzo después de 1975 (Figura 7). Esta variante obedece a que en marzo las mínimas presentan un pico con mayor temperatura cuya frecuencia aumenta después de 1975 como se constata en las Figuras 3.10 a 3.13 de la tesis (Lomelí 2014). Esta anomalía al parecer es una particularidad del nicho.

Temperatura mínima OC 


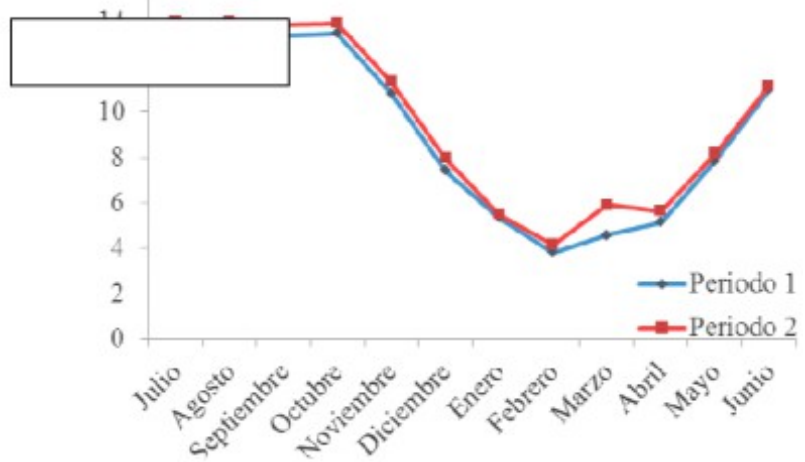

FIGURA 7

Variación de las temperaturas mínimas en la interacción periodos*meses con énfasis en la GSI. Lomelí 2014

\section{Interacción meses ${ }^{*}$ decenas sobre las temperaturas minimas}

Según la Figura 8, el descenso de las temperaturas se da desde octubre y las decenas "tres" se comportan más frías, comportamiento que se extiende hasta diciembre iniciándose una igualación de las decenas para continuar con un comportamiento inverso en febrero donde las decenas "tres" se comportan más tibias extendiéndose hasta junio. Se trata de un proceso un tanto opuesto al de las máximas (Figura 5) con un desfasamiento de los fenómenos de igualación. El pico con incremento de las mínimas en el mes de marzo se confirma pero se extiende hasta junio. El hecho de que ese efecto de calentamiento se de en las mínimas confirma la idea que el fenómeno ocurre en las noches.

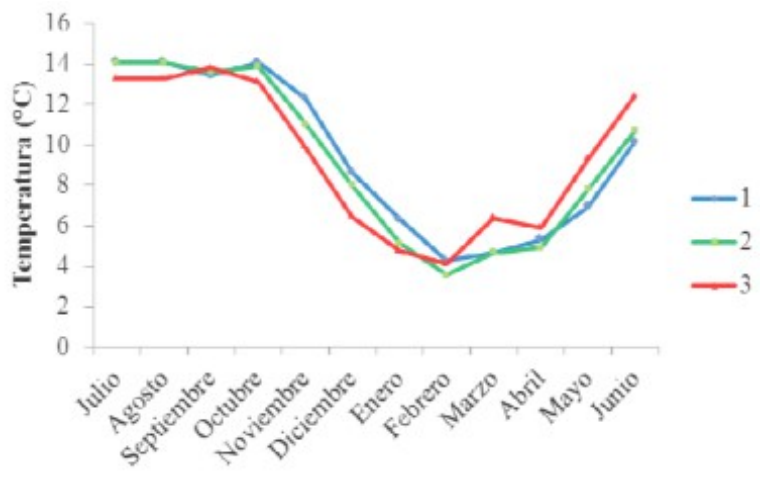

FIGURA 8.

Variación de la temperatura mínima en la interacción meses*decenas con énfasis en la GSI. Lomelí-Sandoval (2014)

Interacción Periodos*Años*Meses respecto a la GSI

En la fuente Lomelí-Sandoval (2014) se examinaron 30 distribuciones de las temperaturas mínimas de las mínimas de los meses de la GSI del periodo 1 (1944-45 a 1973-74) y 30 del periodo 2 (1975-76 a 2004-5). Tres de estas distribuciones correspondientes al periodo 2 (1975-76 a 2004-5) se muestran en la Figura 8. En la imagen de 1996 se presenta un descenso pico en febrero, en la de 1997 ocurren dos enfriamientos pico uno en enero y uno similar en febrero y en 1998 se aprecian tres enfriamientos parecidos: el de enero, el de febrero y el de marzo. En base a la fuente Lomelí-Sandoval (2014) en el Cuadro 1 se muestra la frecuencia de estos eventos antes y después de 1975 anotando dos cifras del año en que se dieron (el año es al que pertenecen los meses enero a junio, por simplicidad se ignora el año precedente al cual pertenecen los meses julio a diciembre) el exponente en las dos cifras del año indica cuantos enfriamientos pico similares ocurrieron en 
esa GSI; donde no hay exponente indica que en esa GSI solo ocurrió un enfriamiento pico. El que aparezcan y aumenten los picos de temperatura mínima similar (Cuadro 1; Figura 8) implica un aumento en el riesgo de la helada sobre todo si se considera que la caña al ser de origen tropical no necesariamente la temperatura debe alcanzar los cero grados para sufrir daño de frio.

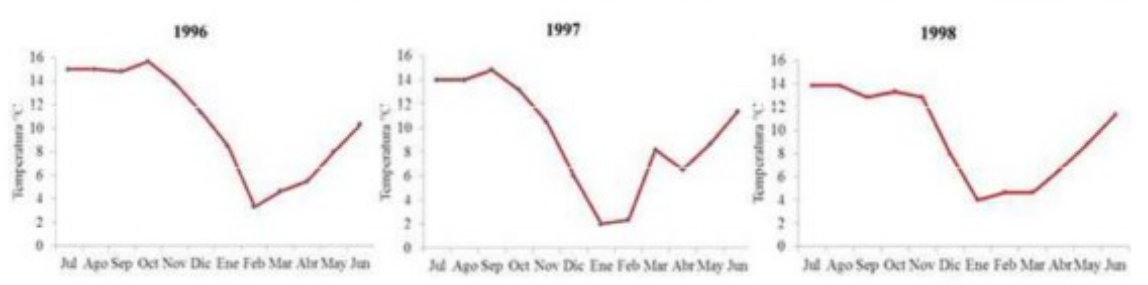

FIGURA 8.

Se muestra la distribución de las temperaturas mínimas de las mínimas mensuales durante la GSI, en 1996 con un descenso pico en febrero, en 1997 con dos similares uno en enero y otro en febrero y en 1998 con tres similares uno en enero otro en febrero y otro en marzo.

Lomelí-Sandoval (2014)

Según el Cuadro 1, (y Cuadro 1 contiruaciön) la frecuencia de descensos pico dobles similares en el periodo 1 fue de dos y en el segundo periodo aumentó y hubo picos no solo dobles sino un caso de cuatro picos en una sola GSI, eso refleja un agrandamiento de los lapsos de enfriamiento y de su variación. También se observa que en febrero del segundo periodo la frecuencia de años con enfriamientos pico aumentó, en marzo disminuyó y en abril aumentó respecto al periodo uno; estas distorsiones en conjunto explican el pico de la decena tres en las Figura 7 y 8.

\section{CUADRO 1.}

Frecuencia de enfriamientos pico de magnitud similar, las dos cifras representan el año en que ocurrieron; sin exponente indica un pico, en los otros los exponentes expresan el número de picos de magnitud similar en la GSI del año de referencia.

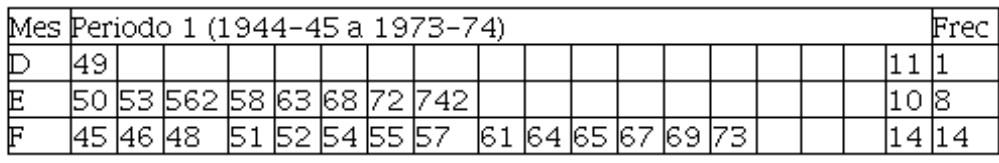

Cuadro 1

\section{CUADRO 1 CONTINUACÓN}

Frecuencia de enfriamientos pico de magnitud similar, las dos cifras representan el año en que ocurrieron; sin exponente indica un pico, en los otros los exponentes expresan el número de picos de magnitud similar en la GSI del año de referencia.

\begin{tabular}{|c|c|c|c|c|c|c|c|c|c|c|c|c|c|c|c|c|c|c|c|}
\hline $\mathrm{M}$ & 59 & 60 & 62 & 66 & 70 & 71 & 742 & & & & & & & & & & & 8 & \\
\hline$A$ & 47 & 562 & 69 & & & & & & & & & & & & & & & 4 & 3 \\
\hline & Perio & odo 2 & $2(1$ & 975 & $-76 a$ & 200 & $4-06$ & & & & & & & & & & & & \\
\hline D & 792 & 994 & & & & & & & & & & & & & & & & 6 & 6 \\
\hline$E$ & 80 & 83 & 86 & 88 & 932 & 972 & 983 & 994 & 012 & & & & & & & & & 17 & 2 \\
\hline F & 75 & 76 & 78 & 792 & 82 & 84 & 85 & 87 & 92 & 94 & 95 & 9 & & 972 & 983 & 994 & 00 & \begin{tabular}{l|l|l}
04 & 24 \\
\end{tabular} & 17 \\
\hline $\mathrm{M}$ & 89 & 90 & 02 & 983 & 99 & & & & & & & & & & & & & \begin{tabular}{|l|l}
10 \\
\end{tabular} & 5 \\
\hline$A$ & 77 & 80 & 86 & 91 & 994 & 1012 & 103 & & & & & & & & & & & 11 & 7 \\
\hline
\end{tabular}

Elaboración propia 


\section{PRECIPITACIÓN DECENAL Gran Sequia Invernal (GSI)}

Gran Sequía Invernal (GSI)

Interacción periodos*meses

En el periodo dos se aprecia una reducción progresiva de lluvia de marzo a mayo respecto al periodo uno atribuible al cambio climático (Figura 9) o sea que el cambio climático está produciendo un re secamiento en ese tiempo.

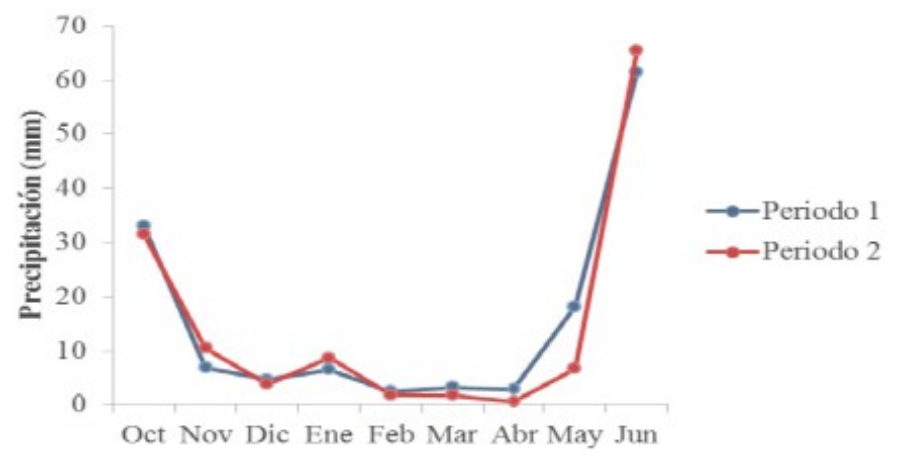

FIGURA 9 (3. 1)

Interacción periodos*meses en relación a la precipitación decenal en el lapso en que ocurre la GSI. Figura 9

\section{Interacción decenas*meses}

En octubre la decena tres es la menos lluviosa y la uno la más lluviosa como reflejo de la reducción de la precipitación a medida que avanza el otoño. En mayo y junio ocurre al revés (Figura 10) dado que en estos meses la lluvia va en aumento por el establecimiento del temporal. La cantidad de lluvia en aumento en la segunda y tercer decena indica que en ese tiempo se establece el temporal; pero a la vez los niveles bajos de lluvia en mayo redundan en el resecamiento a que se hizo referencia (Figura 9). En los meses más secos que van de noviembre a abril las diferencias entre las decenas son mínimas; sin embargo no deja de hacerse notar un mayor resecamiento de marzo a mayo que es influencia de la reducción de lluvia de la Figura 9.

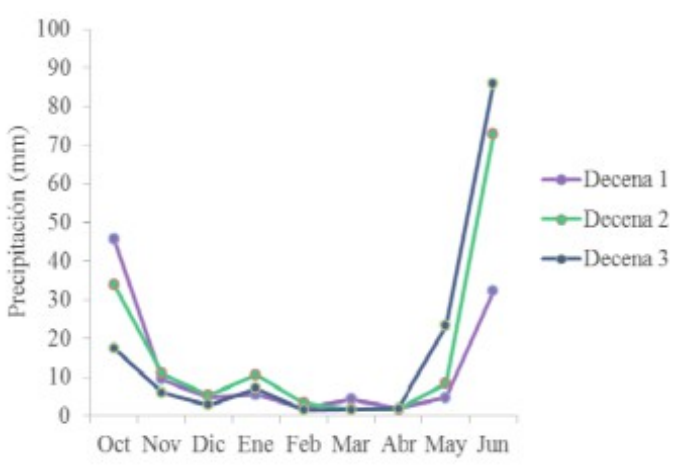

FIGURA 10 (3. 2)

Interacción meses*decenas en relación a la precipitación en el lapso en que ocurre la GSI Figura 10

\section{Sequia Intraestival (SI)}

Interacción periodos*meses

Como se asentó antes la SI es un descenso de lluvia que puede ocurrir de junio a octubre (Reyna, 1970) (Figura 4). En este estudio, en el periodo 2 (después de 1975) para junio y julio da un aumento de la lluvia 
mensual respecto a los mismos meses en el periodo 1 (antes de 1975), en agosto y septiembre los promedio discrepan en mínimas magnitudes (Figura 11); estas diferencias tienen sentido opuesto a las registradas de marzo a mayo para la GSI (Figura 9); aunque de sentido opuesto, ambos efectos son atribuibles al cambio climático. Estos efectos aumentativos de la lluvia que se empiezan a manifestar en junio son reflejo de la acción de otro factor, en este caso relacionado con la ocurrencia de la precipitación.

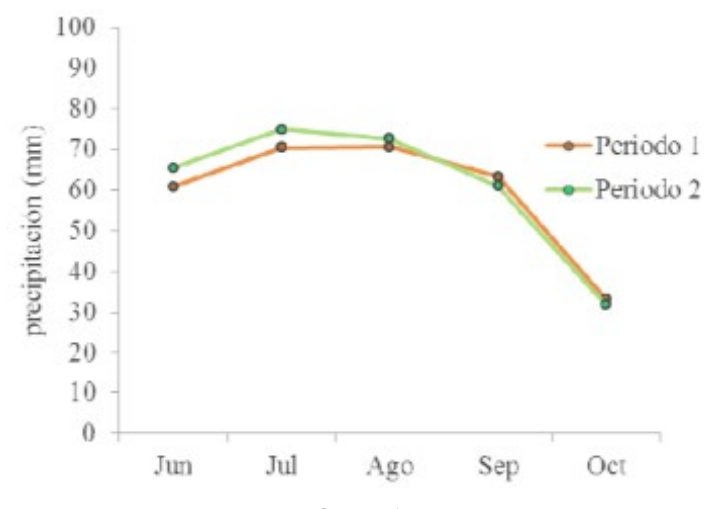

FIGURA 11.

Efecto de la interacción meses* periodos sobre la lluvia, en el lapso en que suele ocurrir la SI. Reyna, 1970

\section{Frecuencia de la SI en los periodos 1 y 2}

El déficit de la lluvia que caracteriza a la SI varía de dos a cinco meses (Reyna, 1970) (Figura 4). Tres tipos de estas variaciones se observaron en este trabajo, mismas que se ilustran la Figura 12. En la imagen de 1977 no hubo SI, en 1992 el déficit se observa entre junio y agosto, en 1968 ocurre entre julio y septiembre; y en la imagen de 1978 se dio entre junio y septiembre. El Cuadro 2 muestra que la SI tuvo menor frecuencia en el periodo 2; en el Cuadro 3 se precisa que de $70 \%$ de incidencia en el periodo 1, cambió a

$50 \%$ en el periodo 2. En México como efecto del cambio climático se aprecia un aumento en la frecuencia e intensidad de los ciclones (Tudela 2011). A este nicho llegan los efectos de los ciclones que ocurren en el occidente de México, mismos que están aumentando su número también; así, en 2014 alcanzó la cifra de 20, ocupando el segundo lugar en la época reciente (Internet 2014). Las frecuencias ilustradas en este trabajo derivan del examen de 60 gráficas de la interacción periodos*años*meses (Lomelí-Sandoval, 2014) relativas al lapso en que suele ocurrir la SI que por ser numerosas no se incluyen aquí. Resumiendo la SI está reduciendo su frecuencia lo que está asociado al aumento de ciclones que impactan el occidente de México en donde está ubicado el nicho de Tula, Jalisco. Este efecto puede explicar el aumento de lluvia de la Figura 11, y relacionarse con el atrapamiento de calor que redundó en aumento de las temperaturas máximas de la Figura 4.

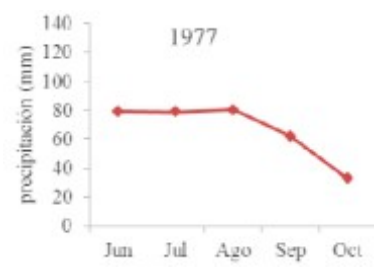

FIGURA 12

Muestra las principales variaciones de la SI: ausente en 1977, presente en julio de 1992, en agosto de 1968 y con duración de dos meses en julio y agosto de 1978. Fuente: Lomelí- Sandoval, 2014) 


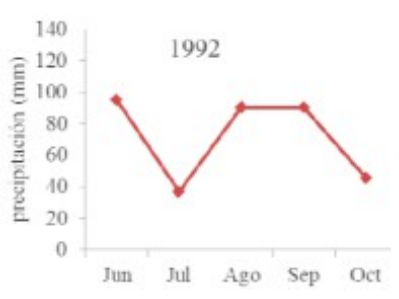

FIGURA 12

Figura 12 Muestra las principales variaciones de la SI: ausente en 1977, presente en julio de 1992, en agosto de 1968 y con duración de dos meses en julio y agosto de 1978. Fuente: Lomelí- Sandoval, 2014)

Lomelí- Sandoval, 2014

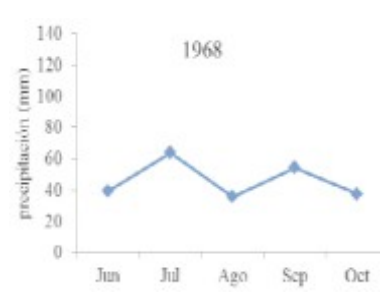

FIGURA 12A.

Figura 12.a Muestra las principales variaciones de la SI: ausente en 1977, presente en julio de 1992, en agosto de 1968 y con duración de dos meses en julio y agosto de 1978. Fuente: Lomelí- Sandoval, 2014) Figura 12

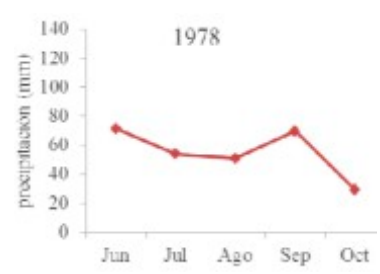

FIGURA 12B.

Figura 12 b. Muestra las principales variaciones de la SI: ausente en 1977, presente en julio de 1992, en agosto de 1968 y con duración de dos meses en julio y agosto de 1978. Fuente: Lomelí- Sandoval, 2014)

Lomelí- Sandoval, 2014)

Cuadro 2. Frecuencia con que se presentó la Sequía Intraestival (SI) en el periodo 1 (Antes de 1975) y en el periodo 2 (Después de 1975), indicando los meses y años en que se dieron los eventos

CUADRO 2.

Frecuencia con que se presentó la Sequía Intraestival (SI) en el periodo 1 (Antes de 1975) y en el periodo 2 (Después de 1975), indicando los meses y años en que se dieron los eventos

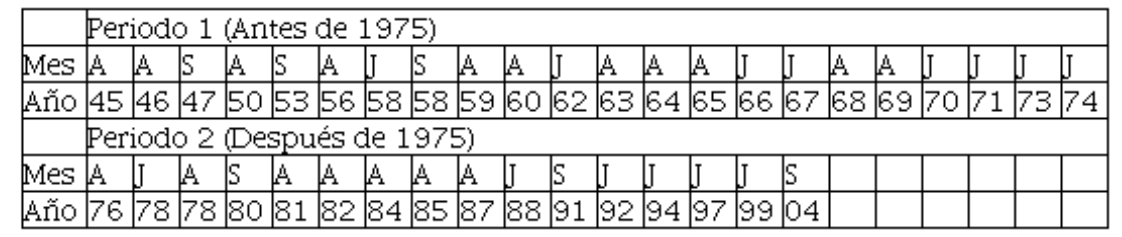

La SI de 1958 fue de dos meses, lo mismo la de 1978. J, julio; A, agosto; S, septiembre Lomelí- Sandoval, 2014) 
CUADRO 3.

Concentración de frecuencias de la SI por meses y por periodos

\begin{tabular}{|l|l|l|l|l|l|l|l|l|}
\hline Mes & $\mathrm{J}$ & $\mathrm{A}$ & $\mathrm{S}$ & $\begin{array}{l}\text { Años } \\
\text { muestreados }\end{array}$ & $\% \mathrm{~J}$ & $\% \mathrm{~A}$ & $\% \mathrm{~S}$ & $\%$ Total \\
\hline $\begin{array}{l}\text { Periodo } \\
1\end{array}$ & 8 & 11 & 3 & 30 & 26.6 & 36.7 & 10.1 & 70 \\
\hline $\begin{array}{l}\text { Periodo } \\
2\end{array}$ & 6 & 7 & 3 & 30 & 20.0 & 23.3 & 10.0 & 50 \\
\hline
\end{tabular}

J, julio; A, agosto; S, septiembre; SI, Sequía Intraestival Cuadro 3

\section{Variaciones de lluvia en enero dentro de la GSI}

Interesó enero porque en el experimento de variedades de caña realizado en 2011-2013 en enero de 2013 se tuvo una semana con lluvias y hubo necesidad de parar el ingenio hasta que se restableciera el nivel de sacarosa dado que decayó el proceso de maduración de las cañas. Examinando las gráficas de lluvia en la GSI del trabajo de Lomelí-Sandoval (2014) se determinó la frecuencia de lluvias en dicho lapso la cual se muestra en la Figura 10. Se observa que Antes de 1975 ocurrió en una ocasión (1967-68) y después de 1975 ha ocurrido en 2079-80, 2091-92 y se observó en 2013 o sea que hay una tendencia a aumentar en el Periodo 2.

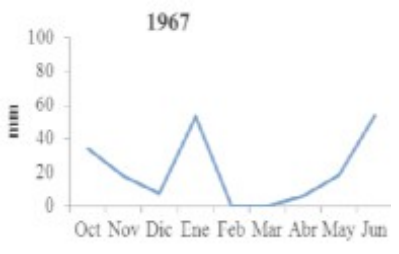

FIGURA 13 (14).

Variaciones de lluvia dentro de la GSI, ocurridas específicamente en el mes de enero. Figura 13

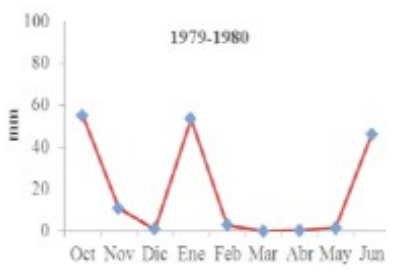

FIGURA 13 (14).

Variaciones de lluvia dentro de la GSI, ocurridas específicamente en el mes de enero. Figura 13

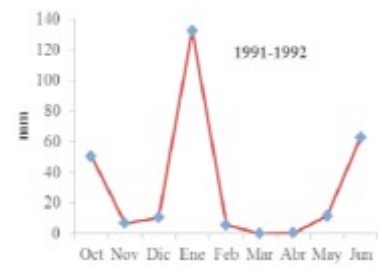

FIGURA 13 (14).

Variaciones de lluvia dentro de la GSI, ocurridas específicamente en el mes de enero.

Figura 13 


\section{Lluvia pico en marzo}

Este evento se presentó en 2012 (Figura 14) en el desarrollo de la plantilla del experimento de variedades de caña bajo sequía realizado cerca de Tamazula, Jalisco (2011-2013). Ocurrió en 1968 y el pico alcanzo 60 mm de lluvia similar al que se presentó en 2012 (Figura 14). De donde se infiere que en general la parte media de la GSI es seca sin diferencias apreciables entre periodo 1 y 2.

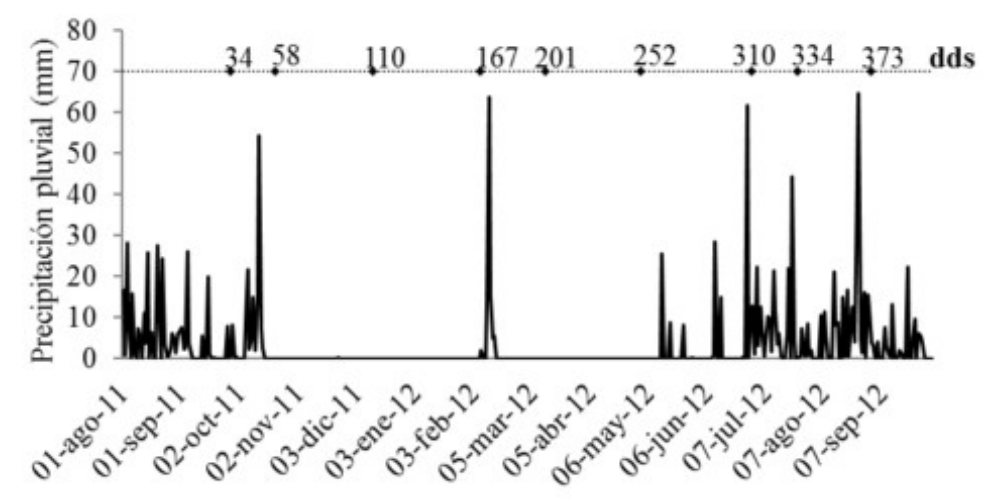

FIGURA 14 (13).

Variaciones de lluvia durante el ciclo de plantilla del experimento de sequía realizado en el área de Tamazula, Jalisco en 2011-12 (Fuente: Lomelí-Sandoval, 2014)

(Lomelí-Sandoval, 2014)

\section{CONCLUSIONES}

\section{Temperaturas máximas}

En este nicho el calentamiento global (Después de 1975) se dio en septiembre y octubre y en abril y mayo se dio un enfriamiento (Figura 4). Esta inversión, puede relacionarse con el ambiente seco que predomina durante la GSI y la condición húmeda durante el verano- otoño.

(Entre decenas)

En el nicho de Tamazula el periodo de más riesgo de golpes de calor para caña se tiene en abril y mayo (Figura 5). El ciclo lluvioso genera una estabilidad térmica en las decenas de los meses de dicho ciclo. Entre las decenas de cada mes se alternan igualaciones e inversiones de las secuencias de temperaturas máximas de cada mes; queda pendiente precisar como la caña se afecta en la fisiología por dichos eventos. Precisar estas dinámicas fue posible por el agrupamiento de los datos mensuales en decenas.

Temperaturas mínimas

Hay un incremento de los grados de las temperaturas mínimas atribuible al cambio climático, sutil después de 1975 y de mayor magnitud después de 1990 (Figura 6); entre los meses del año el incremento se manifiesta en marzo de manera más pronunciada (Figuras 7 y 8 ). Al manifestarse este incremento en las temperaturas mínimas concuerda con la idea que el calentamiento se manifiesta en la noche.

\section{Interacción decenas*meses respecto a máximas y minimas}

El análisis de las temperaturas máximas y mínimas en las decenas dentro de los meses (Figura 5 y 8 ) permitió ver inversiones de los gradientes entre las decenas y ausencias de gradientes (igualación de las máximas o de las mínimas de las decenas) con cierta simetría en las máximas respecto a las mínimas; importantes en la regulación de las resistencias a los factores adversos. Así, el mayor riesgo de heladas ocurre en febrero (Figura 
8) y de golpes de calor en abril y mayo (Figura 5); Los ascensos de temperatura de abril a mayo están asociados a baja humedad, en tales condiciones el ataque de barrenador a la caña de azúcar se intensifica como lo observó Rodríguez (2013).

El que aparezcan y aumenten los picos de temperatura mínima similares después de 1975 (Cuadro 1; Figura 8), atribuibles al cambio climático, implica un aumento en el riesgo de la helada sobre todo si se considera que la caña al ser de origen tropical no necesariamente la temperatura debe alcanzar los cero grados para sufrir daño de frio.

\section{Lluvia decenal}

El cambio climático está generando un resecamiento de marzo a mayo por una reducción de lluvia en el periodo 2 respecto al 1, en el nicho de referencia (Figura 9). En junio y julio meses en que puede ocurrir la SI, en el periodo 2 (después de 1975), se aprecia un aumento de lluvia respecto al periodo 1 (Figura 11), tendencia opuesta a la que se presenta de marzo a mayo (Figura 9) y que refleja la acción de otro factor relacionado, en este caso, con la ocurrencia de la lluvia.

Se detectó una reducción en la frecuencia de la sequía intraestival (SI) después de 1975 asociada al aumento en la frecuencia e intensidad de los ciclones que inciden en el Occidente de México cuyo efecto alcanza el nicho de Tamazula, Jalisco; el aumento de las temperaturas máximas detectado puede estar asociado al aumento de humedad que atrapa en mayor grado la radiación diurna.

El modelo usado y la estrategia de manejo de los factores de variación se consideran bastante eficientes al permitir ver variaciones e interrelaciones indispensables para diseñar esquemas genótecnicos para el uso de la mega diversidad micro regional para afrontar el cambio climático.

La división de los meses en decenas permitió internarse en la anatomía de los cambios termopluviométricos (inversiones e igualaciones de temperaturas y lluvias) y nos deja la tarea de precisar su efecto en la fisiología de la caña.

\section{REFERENCIAS}

Internet. (2014). Temporada de huracanes en el Pacífico de 2014. Weather Channel. 30 de noviembre de 2014.

IPCC, (2001). Climate Change 2001: Synthesis Report. Contribution of Working Groups I, II and III on the Third Assessment Rreport of the Intergovernmental Panel on Climate Change ((Watson R. T., and Core Writing Team (eds)). Cambridge University Press, United Kingdom and New York, N. Y. USA, 398 pp.

Lomelí-Sandoval NA. (2014). Resistencia sequía en caña. Caso de Tamazula, Jalisco. Tesis de Maestra en ciencias. Programa de Recusos Genéticos y Productividad,

Lomelí-Sandoval H, A. Muñoz O, E Solórzano V, S Sánchez D. (2012). Estudio del daño de la helada en caña de azúcar mediante imágenes digitales. In: Preciado RE, I Ramírez R, F Zavala G, ME Pedraza S y Garza G (eds). Memoria de Resúmenes, XXIV Congreso Nacional y IV Internacional de Fitogenética, Monterrey, NL. Pág. 470.

Mosiño AP y E García. (1978). Evaluación de la sequía intraestival en la República Mexicana. Colegio de Postgraduados. Montecillo, México. 15 páginas.

Muñoz OA. (2005). Descifrando la diversidad del maíz de los nichos ecológicos de México. In: A Muñoz O (ed). Centli Maíz. Sda Edición. Colegio de Postgraduados, Motecillo; Méx. Páginas 132-143.

Muñoz-Orozco A, S Miranda C, JA Cuevas S, A Santacruz Vy S Sánchez D. (2009). Resistencias, prehistoria, historia y diferencias de teocintle a maíz. Impresos América, San Vicente Chicoloapan. 109 páginas. México.

Muñoz OA, JL Michel, C Romero, G Peralta V, N LomelíS y H Lomelí S. (2012). Estudio de las temperaturas mínimas en la zona cañera de tala, Jalisco. In: Blanco MF, ÁG Bravo L, J Hernández M, A Lara H, R Magallanes Q, S de J Méndez G y RD Valdez C 
Nora Araceli Lomelí-Sandoval, et al. Cambio Climático a nivel de nicho y caña de azúcar. I Tamazul...

Muñoz-Orozco A. (2014). Siglo XXI: convergencia de factores favorables y adversos, mejoramiento del maíz y capitalización de la mega diversidad. Acta Fitogenética. Sociedad Mexicana de Fitogenética A.C. Volumen 1, Numero 1. Pp. 378-392.

Reyna TT. (1970). Relaciones entre sequía intraestival y algunos cultivos de México. Tesis UNAM, 90 pp.

Rodríguez-Morales JN. (2013). Resistencia a sequía en la caña de azúcar en tlaquiltenango, Morelos. Tesis de Maestro en Ciencias, Genética IREGEP, Colegio de Posgraduados, Montecillo, México. 170 páginas.

Tudela AF. (2011). Impacto del cambio en el sector agropecuario. Subsecretaría de Planeación y Política Ambiental. Segundo Foro Internacional: Administración de Riesgos en el Sector Agropecuario, Cambio Climático, Seguridad Alimentaria y Desarrollo Competitivo. 10 y 11 de octubre de 2011. 